OPEN ACCESS

Edited by:

Hailong Pei,

Soochow University, China

Reviewed by:

Yuen Gao

Michigan State University,

United States

Himanshu Tripathi,

National Institute of Biomedical

Genomics (NIBMG), India

*Correspondence:

Jian Liu

liujian509@hfut.edu.cn

Zhenyu Bai

baizhenyu666@outlook.com

Specialty section:

This article was submitted to Molecular and Cellular Oncology,

a section of the journal

Frontiers in Oncology

Received: 29 October 2019

Accepted: 09 January 2020

Published: 14 February 2020

Citation:

Chen J, Wang L, Wang F, Liu J and

Bai Z (2020) Genomic Identification of RNA Editing Through Integrating Omics Datasets and the Clinical Relevance in Hepatocellular Carcinoma. Front. Oncol. 10:37. doi: 10.3389/fonc.2020.00037

\section{Genomic Identification of RNA Editing Through Integrating Omics Datasets and the Clinical Relevance in Hepatocellular Carcinoma}

\author{
Juan Chen ${ }^{1}$, Lu Wang ${ }^{1}$, Fangbin Wang ${ }^{1}$, Jian Liu ${ }^{1,2 *}$ and Zhenyu Bai ${ }^{3 *}$ \\ ${ }^{1}$ School of Food and Biological Engineering, Hefei University of Technology, Hefei, China, ${ }^{2}$ Engineering Research Center of \\ Bio-Process, Ministry of Education, Hefei University of Technology, Hefei, China, ${ }^{3}$ Department of Laboratory, General \\ Hospital of Pingmei Shenma Medical Group, Pingdingshan, China
}

RNA editing is a widespread post-transcriptional mechanism to introduce single nucleotide changes to RNA in human cancers. Here, we characterized the global RNA editing profiles of 373 hepatocellular carcinoma (HCC) and 50 adjacent normal liver samples from The Cancer Genome Atlas (TCGA) and revealed that most editing events tend to occur in minor percentage of samples with moderate editing degrees (20-30\%). Moreover, these RNA editing prefer to be A-to-I RNA editing in protein coding genes, especially in $3^{\prime} U T R$ regions. Considering the association between DNA mutation and RNA editing, our analysis found that RNA editing maybe a complementary event for DNA mutation of HCC risk genes in HCC patients. We next identified 454 HCC-related editing sites, and many locate on the same genes with the same editing patterns. The functional consequences of editing revealed 2,086 functional editing sites and demonstrated that most editing in coding regions are non-synonymous variations. Furthermore, our results showed that editing in the $3^{\prime} U T R$ regions tend to influence miRNA-target binding, and the editing degree seems to be negatively correlated with gene expression. Finally, we found that $46 \mathrm{HCC}$-related editing sites with consequence are able to distinguish the prognosis differences of HCC patients, suggesting their clinical relevance. Together, our results highlight RNA editing as a valuable molecular resource for investigating HCC mechanisms and clinical treatments.

Keywords: RNA editing, hepatocellular carcinoma, post-transcriptional regulation, bioinformatics, prognosis

\section{INTRODUCTION}

Hepatocellular carcinoma (HCC) is a kind of malignant tumor with high mortality. It ranks third among all cancer-related mortality in the world. About 3\% of the patients with cirrhosis can result in HCC, which is the most serious complication in chronic liver diseases (1). The high mortality rate of HCC is mainly due to it being asymptomatic in the early stage and the lack of effective treatments for even mid-term patients. This poses a great threat to the patient's life and also brings heavy economic burdens to the society and families. Therefore, it is of great significance to clarify the pathogenesis of HCC as soon as possible, and thus formulate more effective strategies for clinical diagnosis and treatment. Previous studies on the pathogenic mechanisms of cancer suggested that DNA mutations are a driving factor in cancer development; however, many HCC tumor samples 
were found to be free of carcinogenic DNA-driven mutations (2), which indicate that other driving events are involved in the occurrence and development of HCC.

RNA editing is an important post-transcriptional modification event, which change genetic information at RNA level and generate results similar to DNA mutations, thus increasing the diversity of transcripts and proteomes (3). The most common type of RNA editing in human cells is the transformation of adenine nucleotides (A) into inosine (I), which was mediated by adenosine deaminase family proteins (ADARs) (4). Because inosine (I) is recognized as guanine $(G)$ nucleotide during translation, it is also called A-to-GRNA editing. In addition to changes in A-to-I (G) RNA editing, human cells also have a small number of other RNA editing types (5). Recent bioinformatics analysis found that RNA editing events are extensive across the human transcriptome (6).

RNA editing in normal cells are associated with adaptive evolution and cell development (7). Conversely, dysfunction in RNA editing systems will have a series of effects on subsequent RNA regulation processes. First, RNA editing in the protein coding region can affect amino acid translation, producing proteins with different structures or functions, and then affecting protein expression activity. For example, the A-to-I hyper-editing in RHOQ transcripts will induce the abnormal elevation of RHOQ protein, and thus promote the invasion and metastasis of cancer cells in colorectal cancer (8). The editing of SLC22A3 transcript can induce the down-expression of SLC22A3 protein, which contributes to the early invasion in familial esophageal squamous cell carcinoma (9). Second, about 95\% of multi-exon genes produce different transcripts through alternative splicing $(10,11)$, which is common in human liver cancer (12). RNA editing occurring in splicing sites or splicing regulatory elements possibly affects the variable splicing processes of RNA. For instance, the hyper-editing on one of the potential branching sites of PTPN6 causes the third intron retention, which is associated with the pathogenesis of acute leukemia (13). Third, genomewide sequencing analysis revealed that most RNA editing sites were located in the non-coding regions of the genome, including the $3^{\prime} \mathrm{UTR}$, intron and intergenic regions, which may influence the regulation of non-coding RNAs, especially miRNA regulation on the $3^{\prime} \mathrm{UTR}$ regions $(14,15)$. These studies indicate that RNA editing events will affect a series of downstream RNA regulation processes, which are closely related to the disease processes.

Recently, the incidence and progression of HCC were found associated with RNA editing events and may further help us reveal the pathogenic mechanisms underlying HCC. Chen et al. suggested that the hyper-editing event of AZIN1 results in a serine-to-glycine substitution at residue 367 of the AZIN1 protein, which may be a potential driver in the pathogenesis of HCC (16). Chan et al. identified an average of 20,007 A-to-I RNA editing events in transcripts by utilizing RNA-seq of three paired HCC and their adjacent non-tumor samples, then validated the expression level of ADARs that are related to editing degrees of FLNB and COPA in a large cohort with microarray analysis. They also found that the expression level of ADARs which mediate A-to-I RNA editing is related to the risk of HCC recurrence (17). Similarly, research on two pairs of HCC patients revealed that BLCAP transcript is hyper-edited, which will enhance the phosphorylation of AKT, MTOR, and MDM2 and inhibit the function of TP53, thus promote cell proliferation and tumor development (18). Another study identified HCC-related RNA editing sites by genomic and transcriptomic analysis of nine pairs of HCC and normal samples (19). However, the number of HCC patients used in these studies is relatively small, and their statistical efficacy is limited. Furthermore, the main concern is the limited effect of ADAR enzymes on RNA editing, and the limited analysis of downstream regulation systems, which RNA editing may affect.

Recently, The Cancer Genome Atlas (TCGA) project provides a large number of omics data of malignant tumors. Han et al. and Paz-Yaacov et al. identified RNA editing sites in multiple cancer types from TCGA, including $\operatorname{HCC}(20,21)$. However, they focused on pan-cancer analysis and only used a part of the HCC samples. Han et al. focused on existing RNA editing sites annotated in the RADAR database. Moreover, they removed all mutation sites annotated in the COSMIC database and the ones that were not matched to specific tumor samples. Thus, they may miss a number of HCC-related RNA editing events. Paz-Yaacov et al. focused on the clinical influence of Alu-specific RNA editing and just considered 30 pairs of HCC cancer and normal samples. Most importantly, the specific changes in downstream RNA regulation system caused by RNA editing were not thoroughly analyzed, and the genome-wide distribution pattern of RNA editing in HCC is not described in both studies. In addition, as they focused on the common pathogenic mechanism of multiple cancer types, many HCC-related RNA editing sites were not presented. We suggested that it should be clearly described whether the RNA editing was observed in cases where DNA mutations are absent in HCC-relevant driver genes.

In our study, we intend to de novo identification of HCCrelated RNA editing sites by integrating multiple omics data with bioinformatics methods, including genomic mutation, transcriptomic variation, and reference single nucleotide polymorphism (SNP) information, using 373 HCC tumor samples and 50 adjacent normals from TCGA. Here, the genomic distribution pattern of RNA editing events was described. We also deeply analyzed the influence of HCC-related RNA editing on downstream regulation system, including the effect on protein translation and miRNA regulation. Based on clinical information of HCC patients, our study further identified new biomarkers for clinical prognosis, which will promote the disclosure of molecular mechanisms of HCC from the perspective view of RNA editing.

\section{MATERIALS AND METHODS}

\section{Data Retrieval}

Pair-end RNA-seq BAM files originating from 373 HCC cancer samples and 50 adjacent normal liver samples were downloaded from the database of Genotypes and Phenotypes ( $\mathrm{dbGaP}$ ) originally from The Cancer Genome Atlas (TCGA, https:// portal.gdc.cancer.gov/) research project (22). Validated RNAseq FASTQ files of HCC cell lines and normal liver samples were downloaded from ArrayExpress (https://www.ebi.ac.uk/ 
arrayexpress/, E-MTAB-4052) (23), including three human normal liver samples and two Huh7 RNA-seq FASTQ datasets. DNA mutation, gene expression, and clinical information datasets were also downloaded from TCGA. Single nucleotide polymorphism (SNP) annotations were downloaded from dbSNP version 137 (24) and the 1000 Genomes Project (25).

Gene annotation of the $3^{\prime} \mathrm{UTR}, 5^{\prime} \mathrm{UTR}$, CDS, and intron regions were downloaded from the UCSC table browser (http:// genome.ucsc.edu/cgi-bin/hgTables) (26). In addition, functional annotation gene sets were downloaded from the MsigDB database (http://software.broadinstitute.org/gsea/msigdb) (27).

We obtained the list of known tumor suppressive genes (TSGs) and oncogenic genes (OGs) from a previous study (28), which integrates mRNA expression, copy number variations $(\mathrm{CNV})$, and DNA mutation information and generated a continuous ranked list for each gene, ranging from more negative (TSGs) to more positive (OGs) with consistent changes across tumors. Here we used a strict score threshold to get OGs (score $\geq 7$ ) and TSGs (score $\leq-7$ ) for further analysis. The statistical significances for the enrichment of OGs and TSGs were calculated by hypergeometric tests. All of the data resources mentioned above are shown in Supplementary Table S1.

\section{De novo Detection of RNA Editing Sites}

First, the downloaded BAM files of the TCGA samples were converted to FASTQ using BEDtools (29), the FASTQ files were aligned to the human reference genome (GRCh38) by STAR with default parameters (--outFilterMultimapScoreRange 1 -sjdbScore 2 -outFilterScoreMinOverLread 0.33 --outFilterMultimapNmax 20 --sjdbOverhang 100) (30). Second, putative RNA editing sites were identified by Genome Analysis Toolkit (GATK4) with default parameters (HaplotypeCaller --gcs-max-retries 20 --heterozygosity 0.001 --max-reads-peralignment-start 50 --min-base-quality-score 10), using uniquely mapped reads after PCR duplicates were removed (31). Third, computational filters for $v c f$ files were applied through five steps: (i) removing DNA mutation sites for each HCC sample; (ii) taking out all known SNPs in dbSNP version 137 or the 1000 Genome Project, and also insertion or deletion sites; (iii) further filtering sites to obtain editing sites with high confidence: if Fisher Strand (FS) $>20$, or Quality by Depth (QD) $<2$, or editing was supported $<2$ reads, or total coverage reads $<10$, these sites were removed, and we required at least $10 \%$ difference between the editing degrees of $90 \%$ quantile and $10 \%$ quantile across all samples; (iv) sites with $100 \%$ editing degree were also filtered, as $100 \%$ editing efficiency is thought to be unrealistic (6); (v) keeping variants detected in at least $1 \%$ of the samples because they are unlikely to be rare variants. Editing degree was defined as the percentage of edited reads among the total mapped reads at a given site (20).

Finally, we restricted editing sites to 46 human chromosomes. To get the exact RNA, which was edited, we used BEDtools to map the editing sites with gene annotation $g t f$ files. If the RNA editing sites were simultaneously mapped to two strands, these sites were further removed. At last, we get 19,431 RNA editing sites for further analysis.
The validated RNA-seq FASTQ datasets were aligned to the human reference genome (GRCh38) by bowtie, processed by GATK4 with default parameters, with no matched DNA mutation datasets. Other pipelines were similar to the aforementioned method.

\section{Identification of HCC-Related RNA Editing Sites}

HCC-related RNA editing sites include HCC gain, HCC loss, and significant dysregulated editing (dys-edit) sites. HCC gain or HCC loss editing sites were defined by Fisher's exact test (Benjamini-Hochberg correction, adjust $p<0.05$ ), with HCC gain editing sites restricted to be no more than $5 \%$ editing sites in normal samples and HCC loss editing sites restricted to be no more than $5 \%$ editing sites in cancer samples. Dys-edit sites were just focused on the editing degree of 50 HCC cancer samples and mapped 50 normal samples, which were determined by two steps: (i) using the paired Student's $t$-test (Benjamini-Hochberg correction, adjust $p<0.2$ and $p<0.01$ ); (ii) restricting editing sites to those having more than 0.25 editing degree change in at least two pairs of normal and cancer samples. The HCC dys-edit sites identified in the above two steps were changed in the same direction (simultaneously hyper-edited or hypo-edited in two steps). Finally, we identified 454 HCC-related RNA editing sites.

\section{Functional Enrichment Analysis for HCC-Related RNA Editing Sites}

First, HCC-related RNA editing sites were mapped to gene name by BEDtools. Then, we performed functional enrichment analysis by hypergeometric test (Benjamini-Hochberg correction, adjust $p<0.05)$. We focused on chemical and genetic perturbations (CGP), reactome pathways and biological processes $(\mathrm{BP})$ of gene ontology (GO) originating from MsigDB.

\section{The Functional Consequence Analysis for RNA Editing Sites}

\section{Protein Coding and Alternative Splicing Change}

To define whether an editing site can change protein translation or alternative splicing, we re-annotated them by ANNOVAR (32).

\section{miRNA-Target Binding Prediction}

RNA editing in the $3^{\prime}$ UTR regions may influence the binding and regulation of miRNAs, including loss of existing miRNA-target regulation and gain of new regulation, or change the binding strength of existing miRNA-target regulation. Therefore, for each RNA editing site in the $3^{\prime} \mathrm{UTR}$ regions, we simultaneously calculated and compared the miRNA binding in both edited and reference $3^{\prime}$ UTR regions. We computationally predicted whether an miRNA binds to the mRNA regions around editing sites using miRanda (33) as described in Hwang et al. (5), which calculates the binding energy to estimate the thermodynamic properties of a predicted duplex and a complementarity score to estimate the mismatch. Briefly, for mRNA as a target sequence, two types of sequences were prepared with flanking regions of editing sites (50 bp upstream and downstream) in all mRNA transcripts in UCSC: the reference sequence and the editing sequence. The mature miRNA sequences were also prepared, which were 
obtained from miRBase (34). The binding energies between miRNA sequence with both a reference and edited mRNA sequences were calculated by miRanda (v3.3a) with default parameters (score $>140$, gap-open penalty set to -4 and gapextend penalty set to -9 ). We next used delta $\mathrm{G}<-14 \mathrm{kcal} / \mathrm{mol}$ as a threshold for free energy of duplex formation to obtain more confident miRNA-target regulations, which were maintained for the following analysis. The comparison was performed for these two types of mRNA sequence, with all the predicted binding pairs of miRNAs and mRNA targets. If miRNA-target relationships just appeared in reference but not in the edited sequence, these were defined as "edited loss" conversely, defined as "edited gain." As for the relationships that both appeared in the reference and edited sequences, if the binding energies changed more than $14 \mathrm{kcal} / \mathrm{mol}$ between the reference and edited sequences, they were defined as "edited change." The "edited gain," "edited loss", and "edited change" constituted the candidate pool of RNA editing sites that may induce miRNA-target regulations to change. Finally, if the editing of an mRNA target induces more than 10 miRNA-target regulation change, this editing site was defined as having an miRNA-target regulation consequence.

\section{Expression-Related RNA Editing Sites}

To define whether RNA editing can influence RNA expression, we calculated the Pearson correlation between editing degree and expression level among samples where both expression and editing degree were measured (editing degree is not 0; Benjamini-Hochberg correction; adjust $p<0.05)$. The normalized gene expression levels of raw read counts were calculated using DESeq2 (35).

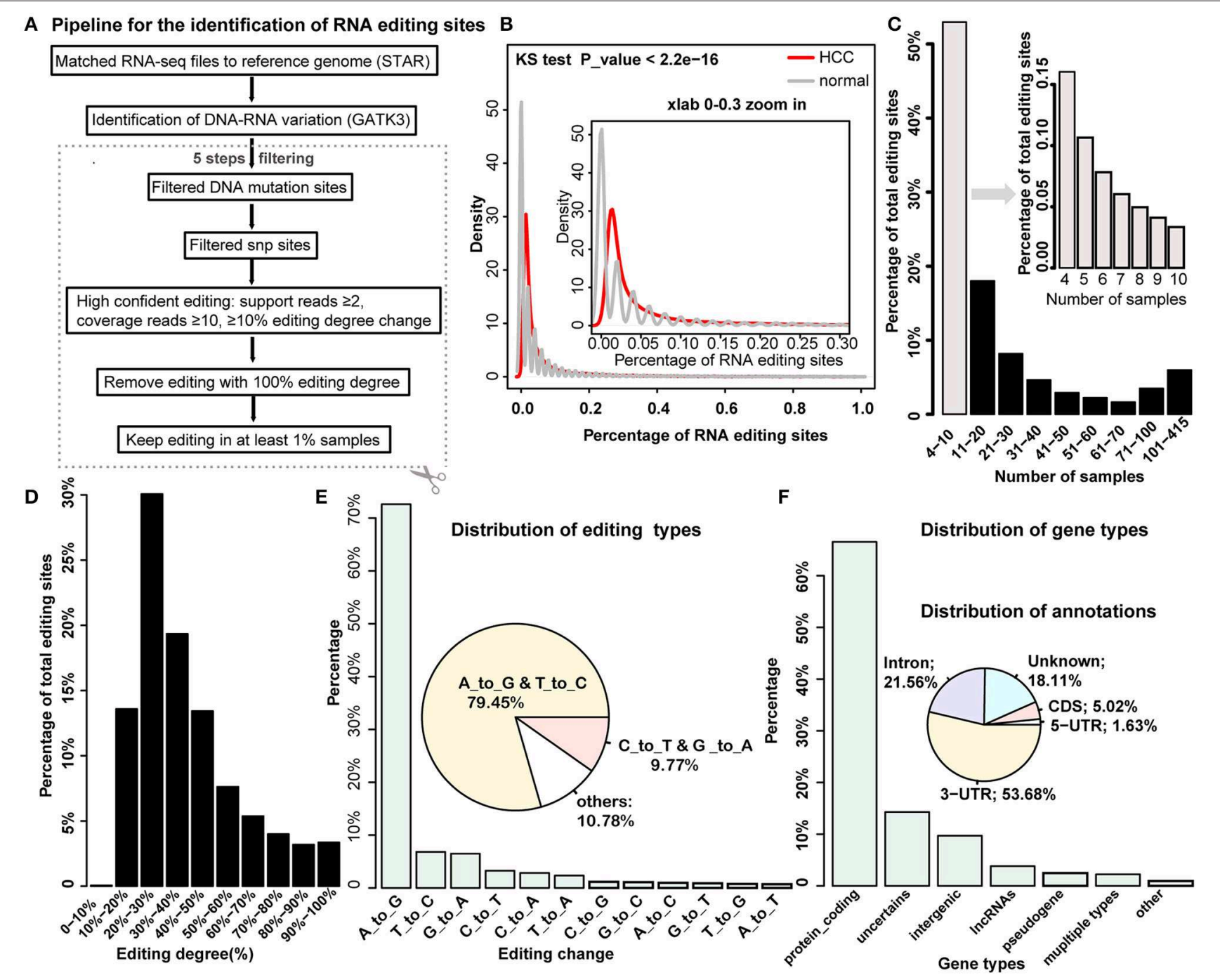

FIGURE 1 | Systemic identification and global properties of RNA editing sites in hepatocellular carcinoma (HCC) and normal samples. (A) The pipeline for the identification of RNA editing site in HCC and normal samples. (B) The distribution of the percentage of RNA editing sites in HCC cancer and normal sample. (C) The distribution of editing percentages across different intervals of samples. (D) The percentage of editing sites across different intervals of editing degrees. (E) The distribution of editing variation types. (F) The distribution of gene types and genomic annotations for identified RNA editing sites. 


\section{Correlation Between Editing Risk Score and Survival}

First, we defined the editing risk score for each HCC clinical sample. Univariate Cox regression analysis was performed to evaluate the association between survival duration and the editing degree of each editing site. A regression coefficient with a plus sign indicated that increased editing degree is associated with an increased risk of survival (risky editing); conversely, a minus sign indicated that an increased editing degree is associated with a decreased risk of survival (protective editing). More specifically, for editing sites, which are located in a gene region, we assigned a risk score to each HCC patient according to a combination of the product of the editing degree and gene expression, weighted by 1 or -1 according to the regression coefficients from the univariate Cox regression analysis mentioned above. The risk score for each patient was calculated as follows:

$$
\text { Risk_Score }=\sum_{i=1}^{n} \beta_{i} * \operatorname{Exp}_{\text {gene }(i)} * \text { Edit }_{i}
$$

where, $\beta_{i}$ is 1 or -1 when the Cox regression coefficient of the editing site $i$ is a positive or negative value, respectively. The $n$ is the number of HCC-related RNA editing sites with consequence that are located in gene regions with an editing degree more than 0 in each sample. Expgene(i) is the expression level of gene in which the editing site $i$ occurs. Edit $t_{i}$ represents the editing degree of the editing site $i$.

To investigate whether risk scores are associated with tumor grades and tumor stages, we use the Wilcoxon rank-sum test. As the sample number with a tumor grade 4 or tumor stage 4 is too small, only 12 and 13 , respectively, we only considered grade 1 , grade 2 , and grade 3 plus (including grade 3 and grade 4) for tumor grades and stage 1, stage 2, and stage 3 plus (including stage 3 and stage 4) for tumor stages. All patients were then classified into high-risk and low-risk groups using the median risk score as the cutoff point. The Kaplan-Meier method was further used to estimate the differences in overall survival time for these two patient groups (log-rank test). Patients having higher risk scores were expected to have poor survival outcomes.

We further focused on three prognostic-related editing sites whose editing degrees are significantly correlated with survival $(p<0.05)$. The risk scores were calculated according to the mathematical formula above. For $\mathrm{HBV} / \mathrm{HCV}$ infected patients or non-alcoholic fatty liver patients, we did a similar analysis mentioned above to obtain a risk score for each sample using three prognostic-related editing sites.

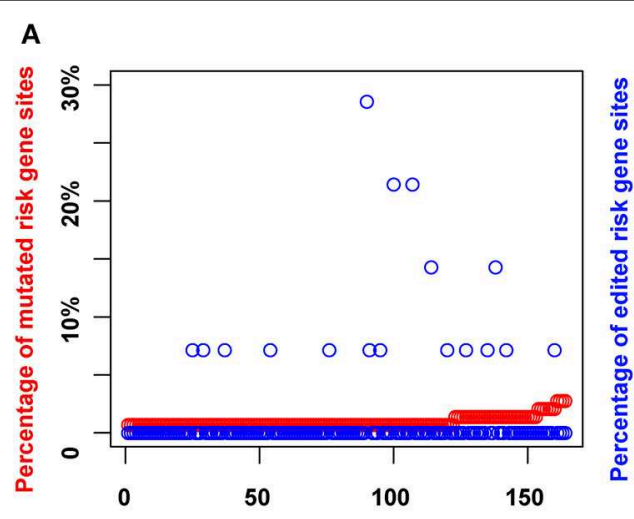

1-164 HCC cases with risk genes mutation
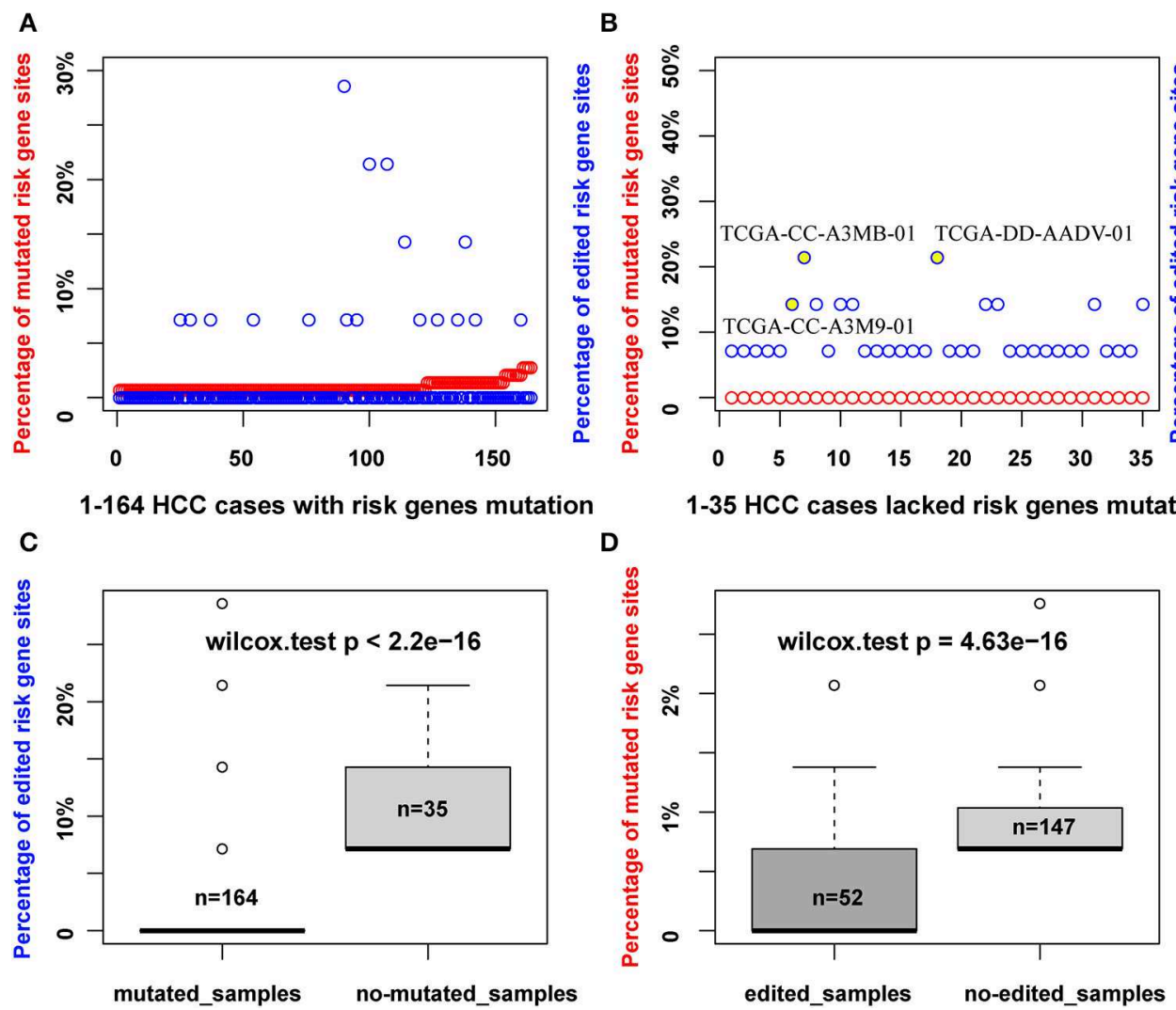

FIGURE 2 | RNA editing maybe a complementary event for DNA mutation of HCC risk genes in HCC patients. (A) The percentage of mutated sites or edited sites in 164 HCC samples with DNA mutation of HCC risk genes (red points, number of mutated sites/total mutated HCC risk sites identified; blue points, number of edited sites/total edited HCC risk sites identified). (B) The percentage of mutated sites or edited sites in $35 \mathrm{HCC}$ samples without DNA mutation of HCC risk genes. (C) The percentage of edited sites account for total edited HCC risk sites identified in mutated and non-mutated HCC samples. (D) The percentage of mutated sites account for total mutated HCC risk sites identified in edited and non-edited samples. 
To determine whether editing risk scores can provide additional predictive power, we performed a multivariate survival analysis using prognostic factors, including gender, age, body mass index (BMI), grade, and stage of HCC patients, along with editing risk score.

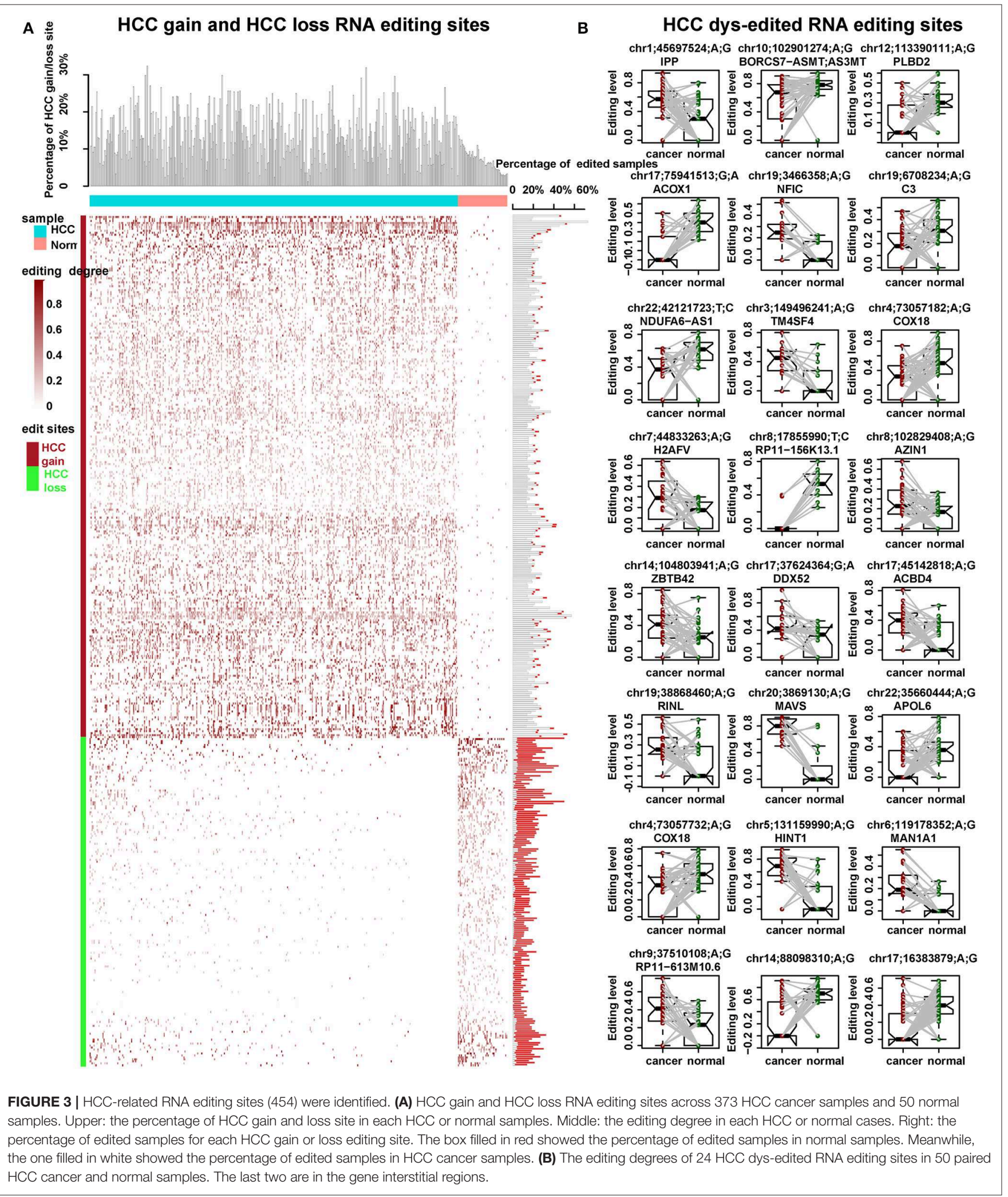




\section{RESULTS}

\section{Global Properties of the Inferred RNA Editing Sites in HCC and Normal Samples}

Several de novo methods for detecting RNA editing were developed recently $(5,6,20)$. Here we combined these methods and proposed a multi-stage method to gradually identify RNA editing sites by integrating DNA mutation and SNP datasets (Figure 1A) (see details in the Methods section). Totally, we obtained 19,431 RNA editing sites for further analysis. In general, HCC tumor samples have a higher percentage of RNA editing sites compared to the normal samples (Figure 1B). More than half of the editing sites occurred in no more than 10 samples (Figure 1C). Most editing sites presented moderate editing degree, where $20-30 \%$ editing degree accounted for the largest

TABLE 1 | HCC-related RNA editing sites.

\begin{tabular}{lccc}
\hline & $\begin{array}{c}\text { Number of editing } \\
\text { sites in } \\
\text { protein-coding } \\
\text { genes }\end{array}$ & $\begin{array}{c}\text { Number of involved } \\
\text { protein-coding } \\
\text { genes }\end{array}$ & $\begin{array}{c}\text { Total number of } \\
\text { RNA editing sites }\end{array}$ \\
\hline HCC gain & 208 & 134 & 264 \\
HCC loss & 93 & 67 & 166 \\
Dys-edited & 19 & 18 & 24 \\
\hline Total & 320 & 213 & 454
\end{tabular}

proportion (Figure 1D). We also found that A-to-IRNA editing accounts for most of the RNA variants in the list, and the following enriched variant types were T-to-C, G-to-A, and Cto-T RNA editing, which was consistent with previous research [Figure 1E; $(5,6)]$. We next annotated these editing sites with gene types and revealed that most of the editing sites are located on protein-coding genes. In addition, a moderate number of RNA editing sites are located in intergenic and lncRNA regions (Figure 1F). Consistent with a previous study, more than half of the editing sites are in the $3^{\prime}$ UTR regions, accounting for $53.68 \%$, and the following are in the intron regions (Figure 1F). Notably, Han et al. reported just a few A-to-I RNA editing in the CDS regions; here we identified about 5\% editing events in the CDS regions, which include many other types of RNA editing. After we restricted to A-to-I RNA editing, the percent decreased to be $1.66 \%$ in the CDS regions (Supplementary Figure S1).

\section{RNA Editing May Be a Complementary Event for DNA Mutation of HCC Risk Genes in HCC Patients}

As RNA editing and DNA mutation have a similar effect to increase the diversity of transcripts in cells, thus we wonder whether there is a connection between these two events. For a given gene region, if it is identified to be mutated, it would not be identified as editing at the same time in our recognition method. However, we can dissect whether there is a correlation between RNA editing and DNA mutation in HCC risk gene sets.

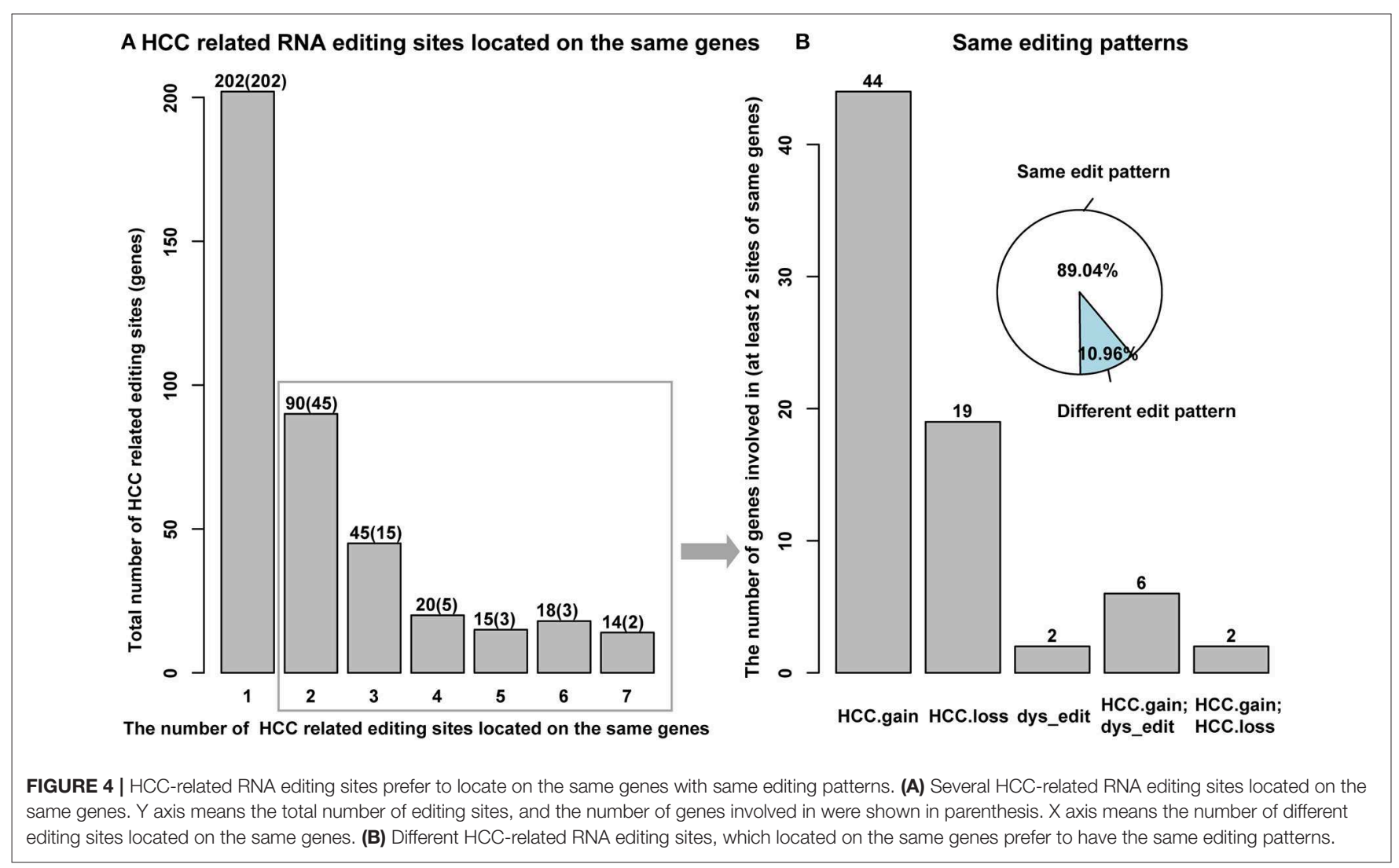


We obtained 33 HCC relevant cancer genes from a previous report (36), which we defined as HCC risk genes, such as TP53, PTEN, and TERT, of which we observed 24 risk genes with DNA mutation (145 mutation sites involved), and six risk genes with RNA editing (14 editing sites involved). HCC patients with DNA mutation (164) and 35 cases lacking DNA mutations in HCC risk genes were observed (Figures $\mathbf{2 A}, \mathbf{B}$ ). It is found that all of these 35 patients that lacked DNA mutation had RNA editing in the HCC risk genes. For example, patient TCGACC-A3M9-01 had no mutated risk genes, but had RNA editing sites in PTEN (chr10:87967417) and UBE2H (chr7:129830735). In patient TCGA-CC-A3MB-01, three sites of RNA editing occurred in the risk gene, TERT (chr5:1262789, chr5:1262852, chr5:1263350), and the same with patient TCGA-DD-AADV-01 (Figure 2B). In addition, we found that the percentage of the edited sites in the HCC risk genes is much higher in patients who lacked DNA mutation compared to patients with DNA mutation in the HCC risk gene regions $(p<2.2 \mathrm{e}-16$, Wilcoxon test) (Figure 2C). Meanwhile, the mutation percentage is much lower in edited patients compared with non-edited patients in HCC

TABLE 2 | Several HCC-related RNA editing sites located on the same genes with the same editing patterns.

\begin{tabular}{|c|c|c|}
\hline Gene & Frequency & HCC-related sites \\
\hline MDM4 & 7 & 7 HCC gain \\
\hline $\mathrm{DHODH}$ & 7 & 7 HCC loss \\
\hline JRK & 6 & 6 HCC gain \\
\hline GINS1 & 6 & 6 HCC gain \\
\hline ALDH2 & 6 & 6 HCC loss \\
\hline SPC24 & 5 & 5 HCC gain \\
\hline SERPINF2 & 5 & 5 HCC gain \\
\hline APC2 & 5 & 5 HCC gain \\
\hline ZNF517 & 4 & 4 HCC gain \\
\hline MOGAT2 & 4 & $4 \mathrm{HCC}$ loss \\
\hline MAVS & 4 & 3 HCC gain; 1 HCC dys-edited \\
\hline HINT1 & 4 & 3 HCC gain; 1 HCC dys-edited \\
\hline ZNF814 & 3 & 3 HCC gain \\
\hline ТTC9C & 3 & 2 HCC gain; 1 HCC dys-edited \\
\hline TRIM56 & 3 & 2 HCC gain; 1 HCC dys-edited \\
\hline $\mathrm{RP} 5-1061 \mathrm{H} 20.4$ & 3 & 3 HCC gain \\
\hline POLR1A & 3 & 3 HCC gain \\
\hline NPLOC4 & 3 & 3 HCC gain \\
\hline MOGAT3 & 3 & 3 HCC gain \\
\hline METTL7A & 3 & 3 HCC gain \\
\hline IPP & 3 & 2 HCC gain; 1 HCC dys-edited \\
\hline HAVCR2 & 3 & 3 HCC gain \\
\hline HAMP & 3 & 3 HCC loss \\
\hline FADS2 & 3 & 3 HCC gain \\
\hline DCAF16 & 3 & 3 HCC gain \\
\hline ADAMTS13 & 3 & 3 HCC loss \\
\hline
\end{tabular}

This table showed genes related to at least three editing sites. risk gene regions ( $p=4.63 \mathrm{e}-16$, Wilcoxon test) (Figure 2D). The observations above implied that RNA editing was a risk factor to HCC as a complementary event for DNA mutation in HCC risk genes.

\section{HCC-Related RNA Editing Prefer to Locate on Liver-Specific Genes}

Chen et al. proved that RNA editing of AZIN1 promotes progression of HCC (16). In our study, by genome-wide identification of RNA editing sites across large RNA-seq samples, we aimed to reveal more HCC-related RNA editing sites. Generally, we identified 454 HCC-related RNA editing sites, including 264 HCC gain, 166 HCC loss, and 24 HCC dysedited sites (Figures 3A,B, Table 1). Notably, a serine-to-glycine substitution at residue 367 of AZIN1 was also identified by our method, which is reported to be hyper-edited in HCC patients compared to normal samples in previous studies (16). Two editing sites of COX18 were hypo-edited in HCC compared with paired normal samples. The most significantly dys-edited RNA editing sites were editing on the ACOX1 (chr17: 75941513, $p=$ 1.15e-05). Notably, ACOX1 was reported to play important roles in cancer development of HCC by stimulating hepatic fatty acid oxidation and $\mathrm{H}_{2} \mathrm{O}_{2}$ accumulation (37).

To further investigate the biological function of these HCCrelated RNA editing sites, we performed functional enrichment analysis for these genes with the HCC-related RNA editing sites (hypergeometric test, adjust $p<0.05$; Supplementary Figure S2, Supplementary Table S2). Our results indicated that HCCrelated editing genes prefer to be liver-specific genes, which were involved in nuclear transport, catabolic and cell cycle processes. The enrichment of liver-specific genes and liver cancer-associated genes suggested that RNA editing can be a potential research area to analyze the mechanism, clinical prevention, and treatment of HCC patients.

\section{HCC-Related Editing Sites Prefer to Locate on the Same Genes With the Same Editing Patterns}

A large percent of HCC-related RNA editing sites were located on gene regions $(88.99 \%, 404 / 454)$ especially for HCC gain and dys-edited sites. Interestingly, 73 genes were shared by at least two HCC-related RNA editing sites and accounted for 50\% (202 editing sites of 404) (Figure 4A, Supplementary Table S3). Notably, we found that different editing sites located on the same genes tend to be with the same HCC editing type, with few exceptions (Figure 4B, Table 2). For example, there are seven HCC-related RNA editing sites located on MDM4; meanwhile, these seven sites are all HCC gain editing patterns. About $89.04 \%$ genes with different editing sites have the same editing patterns, which indicated that these editing sites may have important roles in HCC initiation and development.

\section{The Functional Consequence of RNA Editing Sites}

Previous studies have suggested that RNA editing can be functional and involved in carcinoma initiation and progression 
A

The possible consequences of RNA editing

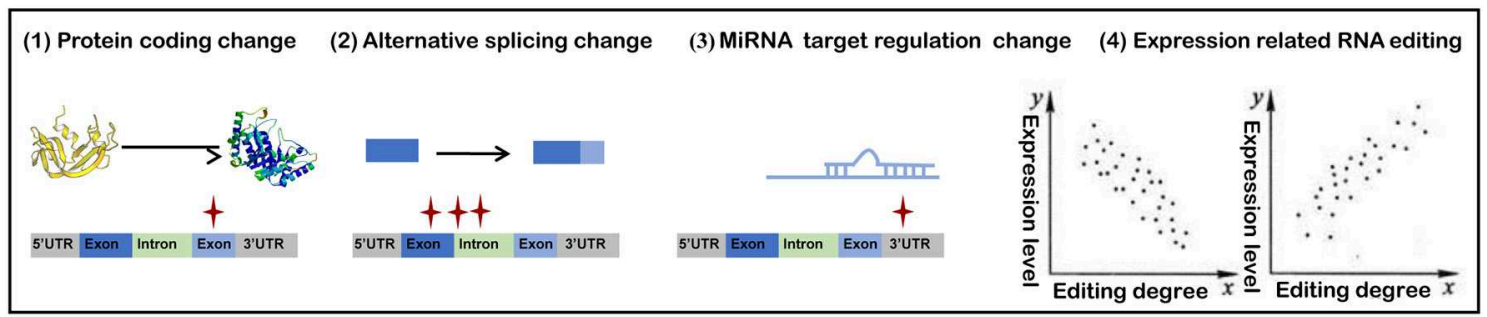

B

The number of RNA Editing sites with possible consequence

\begin{tabular}{ccccccc}
\hline & CDS change & Splicing & \multicolumn{2}{l}{ MiRNA-target } & Expression & Total \\
\hline Total editing & 554 & 30 & 1356 & 163 & 2086 \\
HCC related & 11 & 0 & 26 & 10 & 46 \\
\hline
\end{tabular}

C
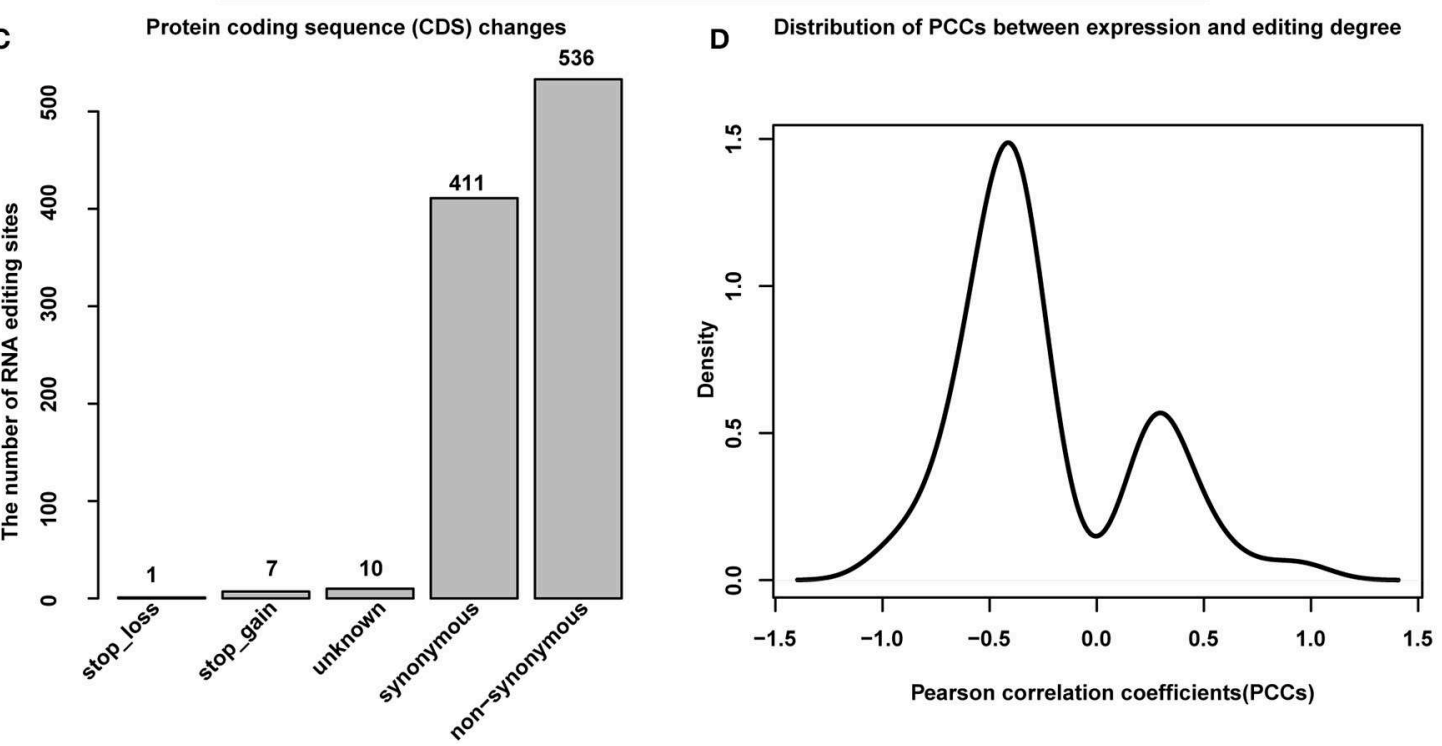

FIGURE 5 | Functional consequence analysis of RNA editing sites. (A) The possible functional consequence of RNA editing, including protein-coding sequence change, alternative splicing change, miRNA-target regulation change, and expression level change. (B) The number of RNA editing sites with possible consequence. (C) The number of RNA editing sites for protein-coding sequence changes. (D) The distribution of Pearson correlation coefficients (PCCs) between editing degrees and the expression of corresponding genes.

by influencing amino acid encoding, alternative splicing, miRNA-target regulation, or expression changing of the corresponding gene $[(38,39)$; Figure 5A]. Totally, we identified 2,064 editing sites with functional consequence, of which 46 were HCC-related (Figure 5B, Supplementary Table S4). For editing in coding sequence (CDS) regions, we identified 554 editing sites with functional change, including one stop loss, seven stop gain, 10 unknown, and 536 non-synonymous (Figures 5B,C), of which 11 editing sites were HCC related. Specifically, the serine-to-glycine substitution at residue 367 of AZIN1 was reported in a previous study; the editing may induce a conformational change and a cytoplasmic-to-nuclear translocation, which will result in tumor initiation and aggressive tumor progression (16). Another example is serine-to-threonine substitution at residue 1,768 of MUC6, which located in a proximate repeat region annotated in the Uniprot database
(40). Importantly, abnormal expression of MUC6 was reported to be associated with many gastrointestinal cancers, such as HCC and cholangiocarcinoma (41). Hence, we supposed that several RNA editing located in important protein domains, which change the properties of the protein, might play roles in the progression of HCC. In terms of alternative splicing, 30 editing sites identified by ANNOVA can induce splicing change, of which no one was HCC related. As more than half of the RNA editing sites located on the $3^{\prime} \mathrm{UTR}$ regions, next, we considered miRNA-target regulation change caused by RNA editing in the $3^{\prime}$ UTR regions. We use miRanda to calculate the binding energies between miRNA sequence and editing sequence (or reference sequence). We identified 1,356 editing sites that affect miRNA-target regulation, of which 26 were HCC related. Among these 26 HCC-related RNA editing sites, the top three affected miRNAs were miR-17-3p, 


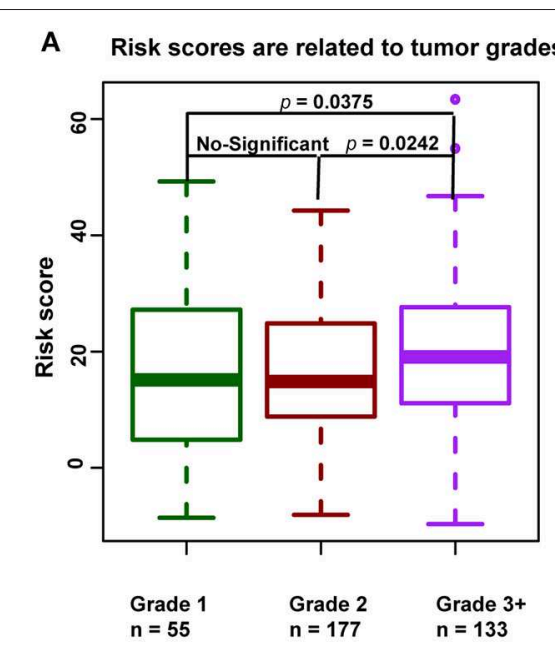

D

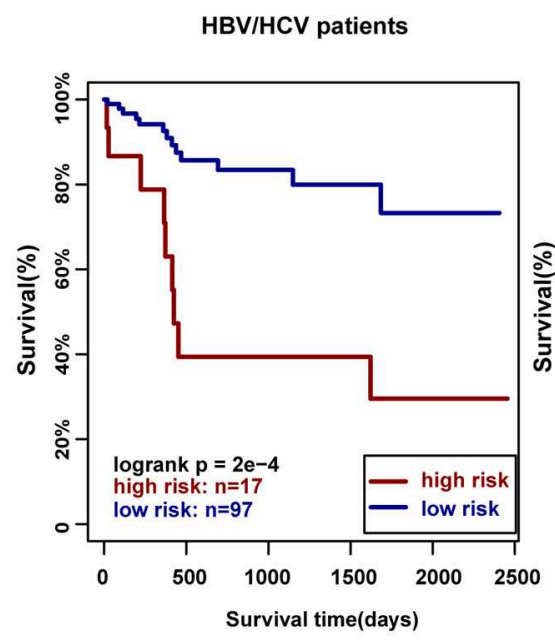

B Risk scores are related to tumor stages

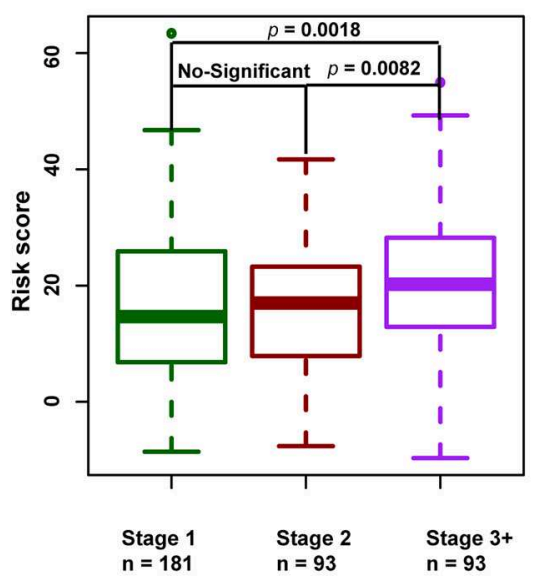

E

Non-Alcoholic Fatty Liver Disease

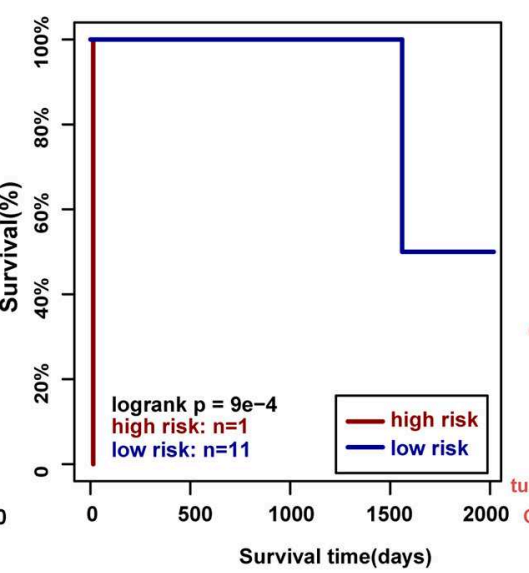

Total HCC patients

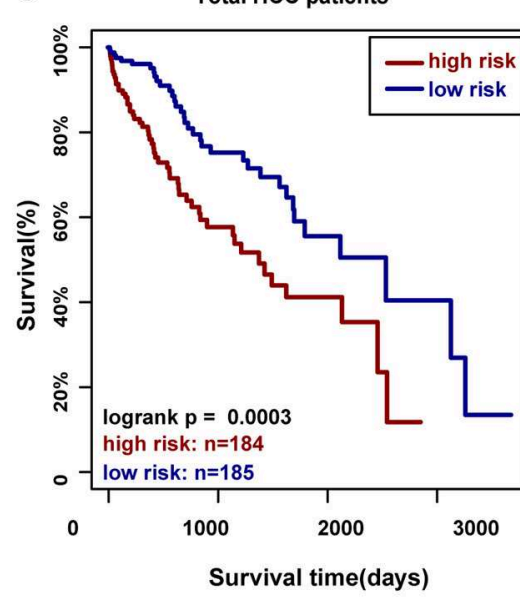

Functional enrichment

F Reactome Pathways

generic transcription pathway

generic transcription pathway
metabolism of proteins

liver specific genes

clim2 targets up

cd5 targets dn

hepatoblastoma classes up

all glucocorticoid therapy $\mathrm{dn}$

breast cancer 17q11 q21 amplicon

BRCA1 pcc network

cervical cancer proliferation cluster

ybx1 targets up
nRAS signaling dn
IL6 deprivation dn

umor differentiated well vs poorly up

Chemical and Genetic Perturbation

$\begin{array}{lllllll}0 & 1 & 2 & 3 & 4 & 5 & 6 \\ & \text { Enrichment(-logFDR) }\end{array}$

FIGURE 6 | The HCC-related RNA editing sites with functional consequence can predict clinical outcomes of HCC patients. (A) Risk scores measured by 46 HCC-related RNA editing sites with functional consequence are related to tumor grades in HCC patients (Wilcoxon rank-sum test). (B) Risk scores measured by 46 HCC-related RNA editing sites with functional consequence are related to tumor stages in HCC patients (Wilcoxon rank-sum test). (C) Risk scores measured by 46 HCC-related RNA editing sites with functional consequence can predict the prognosis in HCC patients. (D) Risk scores measured by three HCC-related prognostic editing sites with functional consequence can predict the prognosis in HBV-/HCV-infected HCC patients. (E) Risk scores measured by three HCC-related prognostic editing sites with functional consequence can predict the prognosis in HCC patients with non-alcoholic fatty liver disease. (F) Functional enrichment of 46 HCC-related RNA editing sites with consequence. The functional gene sets were downloaded from MsigDB, including Chemical and Genetic Perturbation in red and Reactome pathway in purple.

miR-20b-3p, and miR-593-3p (with 11, 7, and 7 target regulation changes induced by HCC-related editing sites, respectively (Supplementary Table S5). Notably, miR-17-3p, miR-20b-3p, and miR-593-3p are widely involved in caner initiation and progression, including HCC (42-46). We observed that the expression levels of 163 RNA editing sites were correlated with the editing degree of corresponding genes, of which 10 were HCC-related editing sites. Moreover, Pearson correlation coefficients (PCCs) tend to be negative values (Figure 5D), which indicated that most of the RNA editing sites might cause downexpression of the corresponding genes.

\section{HCC-Related RNA Editing Sites With Functional Consequence Can Predict Clinical Prognosis}

To further investigate the relationship between RNA editing and HCC clinical characteristics, we first defined the risk score for each clinical sample by integrating the expression levels and editing degrees of HCC-related RNA editing sites with functional consequence, weighted by 1 or -1 according to the regression coefficients from univariate Cox regression analysis (detailed in the Materials and Methods section). As expected, we found that the risk scores measured by 46 HCC-related RNA 
TABLE 3 | The results of multivariate survival analysis of 46 HCC-related RNA editing sites with consequence.

\begin{tabular}{|c|c|c|c|c|}
\hline & \multicolumn{3}{|c|}{ HR (95\% Cl) } & $p$-Value \\
\hline Risk score & \multicolumn{3}{|c|}{$1.03(1.01,1.04)$} & 0.010 ** \\
\hline \multicolumn{5}{|l|}{ Gender } \\
\hline Female & \multicolumn{3}{|c|}{1 (reference) } & \\
\hline Male & \multicolumn{3}{|c|}{$0.90(0.55,1.45)$} & 0.652 \\
\hline Ages & \multicolumn{3}{|c|}{$1.00(1.00,1.00)$} & $0.025^{\star}$ \\
\hline BMI & \multicolumn{3}{|c|}{$1.03(1.00,1.06)$} & 0.097 \\
\hline \multicolumn{5}{|l|}{ Grades } \\
\hline Grade G1 & \multicolumn{3}{|c|}{1 (reference) } & \\
\hline Grade G2 & \multicolumn{3}{|c|}{$1.46(0.65,3.22)$} & 0.369 \\
\hline Grade G3 & \multicolumn{3}{|c|}{$1.74(0.75,4.06)$} & 0.196 \\
\hline Grade G4 & \multicolumn{3}{|c|}{$4.91(1.47,16.39)$} & $0.010^{*}$ \\
\hline \multicolumn{5}{|l|}{ Stages } \\
\hline Stage T1 & \multicolumn{3}{|c|}{1 (reference) } & \\
\hline Stage T2 & \multicolumn{3}{|c|}{$1.31(0.71,2.43)$} & 0.385 \\
\hline Stage T3 & \multicolumn{3}{|c|}{$1.70(0.92,3.13)$} & 0.089 \\
\hline Stage T4 & \multicolumn{3}{|c|}{$3.83(1.45,10.07)$} & $0.007^{\star *}$ \\
\hline \multicolumn{5}{|c|}{ Values of $p<0.05$ were bolded, of which ${ }^{\star} p<0.05$ and ${ }^{* \star} p<0.01$} \\
\hline \multicolumn{5}{|c|}{$\begin{array}{l}\text { TABLE } 4 \text { | Three prognostic HCC-related RNA editing sites with functional } \\
\text { consequence. }\end{array}$} \\
\hline RNA editing & $\begin{array}{l}\text { Gene } \\
\text { types }\end{array}$ & Gene name & Editing type & Function \\
\hline $\begin{array}{l}\text { chr12; } \\
111811793 ; A ; G\end{array}$ & $\begin{array}{l}\text { Protein } \\
\text { coding }\end{array}$ & ALDH2 & HCC loss & miRNA-target \\
\hline $\begin{array}{l}\text { chr9;41929326; } \\
\text { C;T }\end{array}$ & $\begin{array}{l}\text { Protein } \\
\text { coding }\end{array}$ & CNTNAP3B & HCC gain & CDS change \\
\hline $\begin{array}{l}\text { chr9;65675990; } \\
\mathrm{T} ; \mathrm{G}\end{array}$ & $\begin{array}{l}\text { Protein } \\
\text { coding }\end{array}$ & CBWD5 & HCC loss & CDS change \\
\hline
\end{tabular}

editing sites with functional consequence correlated with tumor grades and stages in HCC patients, where higher risk scores in patients implicated higher tumor malignancy (higher tumor grades or tumor stages, Wilcoxon rank-sum test) (Figures 6A,B). We further classified all HCC patients into high-risk and lowrisk groups using the median risk score as the cutoff point. Our analysis suggested that patients having higher risk scores were expected to have poor overall survival times (log-rank test, $p=0.0003$; Figure 6C). The risk score was still significantly associated with patient overall survival (hazard ratio $=1.03, p=$ 0.01 ) in the Cox multivariate analysis, after adjusting for patients' gender, age, BMI, tumor grades, and stages (Table 3), which indicates that these HCC risk RNA editing sites could be potential biomarkers to predict clinical outcomes of HCC patients.

We next identified three prognostic-related editing sites whose editing degrees are significantly correlated with survival time using univariate Cox regression analysis $(p<0.05$; Table 4). The median risk score using these three prognostic editing sites and matched genes can also significantly classify patients into separate groups with different clinical outcomes (log-rank test,
TABLE 5 | The results of multivariate survival analysis of three prognostic HCC-related RNA editing sites with consequence.

\begin{tabular}{|c|c|c|}
\hline & HR (95\% Cl) & $p$-Value \\
\hline Risk score & $1.19(1.07,1.32)$ & $0.001^{* *}$ \\
\hline \multicolumn{3}{|l|}{ Gender } \\
\hline Female & 1 (reference) & \\
\hline Male & $0.84(0.51,1.38)$ & 0.495 \\
\hline Ages & $1.00(1.00,1.00)$ & $0.036^{\star}$ \\
\hline BMI & $1.04(1.01,1.07)$ & $0.009^{\star}$ \\
\hline \multicolumn{3}{|l|}{ Grade } \\
\hline Grade G1 & 1 (reference) & \\
\hline Grade G2 & $1.42(0.64,3.15)$ & 0.390 \\
\hline Grade G3 & $1.88(0.82,4.28)$ & 0.135 \\
\hline Grade G4 & $6.64(2.03,21.76)$ & $0.002^{\star \star}$ \\
\hline \multicolumn{3}{|l|}{ Stage } \\
\hline Stage T1 & 1 (reference) & \\
\hline Stage T2 & $1.12(0.60,2.09)$ & 0.719 \\
\hline Stage T3 & $1.59(0.87,2.91)$ & 0.132 \\
\hline Stage T4 & $3.24(1.20,8.69)$ & $0.020^{*}$ \\
\hline
\end{tabular}

Values of $p<0.05$ were bolded, of which ${ }^{*} p<0.05$ and ${ }^{* *} p<0.01$.

$p=0.03$, data not shown). After adjusting for patients' gender, age, BMI, tumor grades, and stages, the risk score still has predictive power (hazard ratio $=1.19, p=0.001$; Table 5). As $\mathrm{HBV} / \mathrm{HCV}$ infection, alcoholic consumption, and non-alcoholic fatty liver diseases are the three main risk factors to HCC patients, we next examined the predictive power of these three editing sites with these risk factors. We found that these three prognosticrelated editing sites can be used to predict the clinical outcomes for $\mathrm{HBV}-/ \mathrm{HCV}$-infected and non-alcoholic fatty liver disease patients (log-rank $p$-values were $2 \mathrm{e}-4$ and $9 \mathrm{e}-4$, respectively) (Figures 6D,E), but not alcoholic consumption patients (data not shown).

Furthermore, we dissected the function of these 46 HCCrelated RNA editing sites with functional consequence. It is shown that they were widely involved in three reactome pathways: "metabolism of proteins," "generic transcription pathway," and "cell cycle" (Figure 6F, Supplementary Table S6). The functional enrichment analysis for chemical and genetic perturbation datasets indicated that they were enriched in tumor differentiation, nRAS signaling pathway, and liver cancerassociated genes.

\section{DISCUSSION}

HCC is a complex disease with poor prognosis and affected by multiple genetic alterations. DNA mutations are the most widely investigated driver events in all cancer types, including HCC. However, the mutation events are not presented in all HCC patients. Furthermore, we found that $98.51 \%$ mutation sites occurred in only a single HCC patient. Similar to the consequence of DNA mutation, RNA editing can also produce 
nucleotide variations at the RNA level. Importantly, the RNA editing seems to be a more sophisticated regulation, as the editing degree can be $0-100 \%$, while DNA mutation should be mutation or not ( 0 or 1 ). Here we systematically identified RNA editing sites by integrating DNA mutation and SNP datasets, and found that HCC samples have a significantly high percentage of RNA editing sites compared with normal samples. We also observed RNA editing in 35 cases that lacked DNA mutations in HCC risk genes, and more edited risk genes occurred in mutated patients compared with patients who do not have any DNA mutation in the HCC risk gene. Thus, we proposed that RNA editing may be a risk factor to HCC, as a complementary event for DNA mutation in HCC risk genes.

To further characterize the inferred RNA editing sites in our study, we compared the overlap of A-to-IRNA editing sites in gene regions identified by our study and the editing sites collected in the database of RADAR (Supplementary Figure S3). We found that most A-to-IRNA editing sites were in the RADAR database and accounts for $87.11 \%(9,565 / 10,981)$. Furthermore, we re-identified RNA editing sites in three human normal liver tissues and two HCC cell line samples, and found that $38.11 \%$ HCC-related RNA editing sites were edited in at least one sample (173/454), and 39.13\% HCC-related editing sites with functional consequence were identified (18/46). These results indicated that RNA editing sites identified by our research were authentic, which can be used for further analysis. Next, HCC-related RNA editing sites were identified by comparing editing in HCC cancer samples and normal samples. Functional annotation showed that HCC-related editing sites prefer to be liver-specific genes, which suggested the important roles of RNA editing in the development of HCC.

Previous studies focused on the effect of ADAR enzymes on RNA editing. Here we addressed another important question on whether these editing sites have an effect on the downstream regulation system. By considering the influence of RNA editing on coding sequence change, alternative splicing, miRNA-target regulation, and expression change, we identified 2,064 editing sites with functional consequence, which accounts for $10.62 \%$ of the total RNA editing sites $(2,064 / 19,431)$. This percentage indicates that RNA editing events may play other important roles, such as the alteration of RNA-binding abilities, which were induced by RNA structure change, which was, in turn, induced by editing. Notably, we found a number of editing sites with miRNA-target regulation changes. More were identified when we set lower thresholds (Supplementary Table S7). If we defined at least one miRNA relationship change by RNA editing, 90.81\% editing sites in the $3^{\prime} \mathrm{UTR}$ regions can induce miRNAtarget regulation change $(7,651 / 8,425)$. Therefore, we should pay more attention to the effect of miRNA-target regulation change induced by RNA editing. Importantly, in regard to the tumor suppressive genes (TSGs) and the oncogenic genes (OGs) reported (28), we demonstrated that these functional HCCrelated editing genes were significantly enriched in OGs, but not TSGs (data not shown), and it remains true when we set lower thresholds to identify editing sites with miRNA-target regulation changes (Supplementary Table S7).

Importantly, we found 46 HCC-related RNA editing sites with functional consequence that can be used to predict the clinical outcome in HCC patients. In addition, they have independent predictive power after considering the gender, age, BMI, tumor grades, and stages. We identified three clinical prognostic-related editing sites, which can also provide predictive values in $\mathrm{HBV}-/ \mathrm{HCV}$-infected patients and non-alcoholic fatty liver disease patients, including editing sites in ALDH2 (chr12:111811793, A-to-I RNA editing), CNTNAP3B (chr9:41929326, C-to-T RNA editing), and CBWD5 (chr9:65675990, T-to-G RNA editing), of which ALDH2 is an HCC-related editing gene with high frequency (six HCC loss editing sites locates on the ALDH2 gene region). Interestingly, multiple studies revealed that ALDH2 is highly correlated with the pathogenic mechanism, risk, and survival of liver cancer patients, including HCC (47-50). Functional annotation suggested that these HCC-related editing sites with functional consequence are widely involved in liver cancer-associated genes, especially tumor suppressive genes, and cancer-associated pathways. Therefore, we assume that some RNA editing events may be "driver events" that promote cancer initiation and progression, as well as play a critical role in clinical survival in cancer patients.

\section{DATA AVAILABILITY STATEMENT}

The datasets analyzed in this study can be found in the database of Genotypes and Phenotypes (dbGaP) originated from The Cancer Genome Atlas (TCGA, https://portal.gdc.cancer. gov/) and ArrayExpress (https://www.ebi.ac.uk/arrayexpress/, EMTAB-4052).

\section{AUTHOR CONTRIBUTIONS}

$\mathrm{ZB}$ and JL conceived the study. $\mathrm{ZB}$ and JC processed the sequencing data. LW and FW analyzed the data. JC wrote the manuscript.

\section{FUNDING}

This work was supported by the National Natural Science Foundation of China (Grant Nos. 31801114, 31471320, and 31671485), Natural Science Foundation of Anhui Province (Grant No. 1908085QC98), and the Fundamental Research Funds for the Central Universities (Grant No. PA2018GDQT0015).

\section{SUPPLEMENTARY MATERIAL}

The Supplementary Material for this article can be found online at: https://www.frontiersin.org/articles/10.3389/fonc. 2020.00037/full\#supplementary-material 


\section{REFERENCES}

1. Torre LA, Bray F, Siegel RL, Ferlay J, Lortet-Tieulent J, Jemal A. Global cancer statistics, 2012. CA Cancer J Clin. (2015) 65:87-108. doi: 10.3322/caac.21262

2. Huang J, Deng Q, Wang Q, Li KY, Dai JH, Li N, et al. Exome sequencing of hepatitis B virus-associated hepatocellular carcinoma. Nat Genet. (2012) 44:1117-21. doi: 10.1038/ng.2391

3. Peng X, Xu X, Wang Y, Hawke DH, Yu S, Han L, et al. A-to-I RNA editing contributes to proteomic diversity in cancer. Cancer Cell. (2018) 33:817-28.e7. doi: 10.1016/j.ccell.2018.03.026

4. Nishikura K. Functions and regulation of RNA editing by ADAR deaminases. Annu Rev Biochem. (2010) 79:321-49. doi: 10.1146/annurev-biochem-060208-105251

5. Hwang T, Park CK, Leung AKL, Gao Y, Hyde TM, Kleinman JE, et al. Dynamic regulation of RNA editing in human brain development and disease. Nat Neurosci. (2016) 19:1093-9. doi: 10.1038/nn.4337

6. Peng Z, Cheng Y, Tan BCM, Kang L, Tian Z, Zhu Y, et al. Comprehensive analysis of RNA-Seq data reveals extensive RNA editing in a human transcriptome. Nat Biotechnol. (2012) 30:253-60. doi: 10.1038/nbt.2122

7. Yu Y, Zhou H, Kong Y, Pan B, Chen L, Wang H, et al. The landscape of A-to-I RNA editome is shaped by both positive and purifying selection. PLoS Genet. (2016) 12:e1006191. doi: 10.1371/journal.pgen.1006191

8. Han S-W, Kim H-P, Shin J-Y, Jeong E-G, Lee W-C, Kim KY, et al. RNA editing in RHOQ promotes invasion potential in colorectal cancer. J Exp Med. (2014) 211:613-21. doi: 10.1084/jem.20132209

9. Fu L, Qin Y-R, Ming X-Y, Zuo X-B, Diao Y-W, Zhang L-Y, et al. RNA editing of SLC22A3 drives early tumor invasion and metastasis in familial esophageal cancer. Proc Natl Acad Sci USA. (2017) 114:E4631-40. doi: 10.1073/pnas.1703178114

10. Pan Q, Shai O, Lee LJ, Frey BJ, Blencowe BJ. Deep surveying of alternative splicing complexity in the human transcriptome by high-throughput sequencing. Nat Genet. (2008) 40:1413-5. doi: 10.1038/ng.259

11. Li S, Zhang J, Huang S, He X. Genome-wide analysis reveals that exon methylation facilitates its selective usage in the human transcriptome. Brief Bioinform. (2018) 19:754-64. doi: 10.1093/bib/bbx019

12. Li S, Hu Z, Zhao Y, Huang S, He X. Transcriptome-wide analysis reveals the landscape of aberrant alternative splicing events in liver cancer. Hepatology. (2019) 69:359-75. doi: 10.1002/hep.30158

13. Beghini A, Ripamonti CB, Peterlongo P, Roversi G, Cairoli R, Morra E, et al. RNA hyperediting and alternative splicing of hematopoietic cell phosphatase (PTPN6) gene in acute myeloid leukemia. Hum Mol Genet. (2000) 9:2297-304. doi: 10.1093/oxfordjournals.hmg.a018921

14. Hundley HA, Bass BL. ADAR editing in double-stranded UTRs and other noncoding RNA sequences. Trends Biochem Sci. (2010) 35:377-83. doi: 10.1016/j.tibs.2010.02.008

15. Gong J, Wu Y, Zhang X, Liao Y, Sibanda VL, Liu W, et al. Comprehensive analysis of human small RNA sequencing data provides insights into expression profiles and miRNA editing. RNA Biol. (2014) 11:1375-85. doi: 10.1080/15476286.2014.996465

16. Chen L, Li Y, Lin CH, Chan THM, Chow RKK, Song Y, et al. Recoding RNA editing of AZIN1 predisposes to hepatocellular carcinoma. Nat Med. (2013) 19:209-216. doi: 10.1038/nm.3043

17. Chan THM, Lin CH, Qi L, Fei J, Li Y, Yong KJ, et al. A disrupted RNA editing balance mediated by ADARs (Adenosine DeAminases that act on RNA) in human hepatocellular carcinoma. Gut. (2014) 63:832-43. doi: 10.1136/gutjnl-2012-304037

18. Hu X, Wan S, Ou Y, Zhou B, Zhu J, Yi X, et al. RNA overediting of BLCAP contributes to hepatocarcinogenesis identified by wholegenome and transcriptome sequencing. Cancer Lett. (2015) 357:510-9. doi: 10.1016/j.canlet.2014.12.006

19. Kang L, Liu X, Gong Z, Zheng H, Wang J, Li Y, et al. Genome-wide identification of RNA editing in hepatocellular carcinoma. Genomics. (2015) 105:76-82. doi: 10.1016/j.ygeno.2014.11.005

20. Han L, Diao L, Yu S, Xu X, Li J, Zhang R, et al. The genomic landscape and clinical relevance of A-to-I RNA editing in human cancers. Cancer Cell. (2015) 28:515-28. doi: 10.1016/j.ccell.2015.08.013

21. Paz-Yaacov N, Bazak L, Buchumenski I, Porath HT, Danan-Gotthold $\mathrm{M}$, Knisbacher BA, et al. Elevated RNA editing activity is a major contributor to transcriptomic diversity in tumors. Cell Rep. (2015) 13:267-76. doi: 10.1016/j.celrep.2015.08.080

22. Chang K, Creighton C, Davis C, Donehower L, Drummond J, Wheeler D, et al. The Cancer Genome Atlas Pan-Cancer analysis project. Nat Genet. (2013) 45:1113-20. doi: 10.1038/ng.2764

23. Rudolph KLM, Schmitt BM, Villar D, White RJ, Marioni JC, Kutter C, et al. Codon-driven translational efficiency is stable across diverse mammalian cell states. PLoS Genet. (2016) 12:e1006024. doi: 10.1371/journal.pgen.1006024

24. Sherry ST. dbSNP: the NCBI database of genetic variation. Nucleic Acids Res. (2001) 29:308-11. doi: 10.1093/nar/29.1.308

25. Altshuler DM, Durbin RM, Abecasis GR, Bentley DR, Chakravarti A, Clark AG, et al. An integrated map of genetic variation from 1,092 human genomes. Nature. (2012) 491:56-65. doi: 10.1038/nature11632

26. Rosenbloom KR, Armstrong J, Barber GP, Casper J, Clawson H, Diekhans M, et al. The UCSC genome browser database: 2015 update. Nucleic Acids Res. (2015) 43:D670-81. doi: 10.1093/nar/gku1177

27. Liberzon A. A description of the molecular signatures database (MSigDB) web site. Methods Mol Biol. (2014) 1150:153-60. doi: 10.1007/978-1-49390512-6

28. Hamilton MP, Rajapakshe K, Hartig SM, Reva B, McLellan MD, Kandoth $\mathrm{C}$, et al. Identification of a pan-cancer oncogenic microRNA superfamily anchored by a central core seed motif. Nat Commun. (2013) 4:2730. doi: $10.1038 /$ ncomms 3730

29. Quinlan AR, Hall IM. BEDTools: a flexible suite of utilities for comparing genomic features. Bioinformatics. (2010) 26:841-2. doi: 10.1093/bioinformatics/btq033

30. Dobin A, Davis CA, Schlesinger F, Drenkow J, Zaleski C, Jha S, et al. STAR: Ultrafast universal RNA-seq aligner. Bioinformatics. (2013) 29:15-21. doi: 10.1093/bioinformatics/bts635

31. Van der Auwera GA, Carneiro MO, Hartl C, Poplin R, del Angel G, LevyMoonshine A, et al. From fastQ data to high-confidence variant calls: the genome analysis toolkit best practices pipeline. Curr Protoc Bioinformatics. (2013) 43:11.10.1-11.10.33. doi: 10.1002/0471250953.bi1110s43

32. Wang K, Li M, Hakonarson H. ANNOVAR: Functional annotation of genetic variants from high-throughput sequencing data. Nucleic Acids Res. (2010) 38:1-7. doi: 10.1093/nar/gkq603

33. Enright AJ, John B, Gaul U, Tuschl T, Sander C, Marks DS. MicroRNA targets in Drosophila. Genome Biol. (2003) 5:R1. doi: 10.1186/gb-2003-5-1-r1

34. Griffiths-Jones S, Saini HK, Van Dongen S, Enright AJ. miRBase: tools for microRNA genomics. Nucleic Acids Res. (2008) 36:154-8. doi: 10.1093/nar/gkm952

35. Love MI, Huber W, Anders S. Moderated estimation of fold change and dispersion for RNA-seq data with DESeq2. Genome Biol. (2014) 15:550. doi: 10.1186/s13059-014-0550-8

36. Zender L, Villanueva A, Tovar V, Sia D, Chiang DY, Llovet JM. Cancer gene discovery in hepatocellular carcinoma. J Hepatol. (2010) 52:921-9. doi: 10.1016/j.jhep.2009.12.034

37. Yeldandi AV, Rao MS, Reddy JK. Hydrogen peroxide generation in peroxisome proliferator-induced oncogenesis. Mutat Res. (2000) 448:159177. doi: $10.1016 / \mathrm{S} 0027-5107(99) 00234-1$

38. $\mathrm{Xu} \mathrm{X}$, Wang $\mathrm{Y}$, Liang $\mathrm{H}$. The role of A-to-I RNA editing in cancer development. Curr Opin Genet Dev. (2018) 48:51-6. doi: 10.1016/j.gde.2017.10.009

39. Qi L, Chan THM, Tenen DG, Chen L. RNA editome imbalance in hepatocellular carcinoma. Cancer Res. (2014) 74:1301-6. doi: 10.1158/0008-5472.CAN-13-3485

40. Bateman A, Martin MJ, O’Donovan C, Magrane M, Apweiler R, Alpi E, et al. UniProt: a hub for protein information. Nucleic Acids Res. (2015) 43:D204-12. doi: 10.1093/nar/gku989

41. Kasprzak A, Adamek A. Mucins: the old, the new and the promising factors in hepatobiliary carcinogenesis. Int J Mol Sci. (2019) 20:1288. doi: 10.3390/ijms20061288

42. Awan FM, Naz A, Obaid A, Ikram A, Ali A, Ahmad J, et al. MicroRNA pharmacogenomics based integrated model of miR-17-92 cluster in sorafenib resistant HCC cells reveals a strategy to forestall drug resistance. Sci Rep. (2017) 7:11448. doi: 10.1038/s41598-017-11943-1

43. Shan SW, Fang L, Shatseva T, Rutnam ZJ, Yang X, Du WW, et al. Mature miR$17-5 \mathrm{p}$ and passenger miR-17-3p induce hepatocellular carcinoma by targeting 
PTEN, GalNT7 and vimentin in different signal pathways. J Cell Sci. (2013) 126:1517-30. doi: $10.1242 /$ jcs. 122895

44. Guo J, Xiao Z, Yu X, Cao R. miR-20b promotes cellular proliferation and migration by directly regulating phosphatase and tensin homolog in prostate cancer. Oncol Lett. (2017) 14:6895-900. doi: 10.3892/ol. 2017.7041

45. Luo $\mathrm{Y}, \mathrm{He}$ J, Tao X, Wang H, Fang Q, Guo S, et al. miR-20b negatively regulates VEGF expression by targeting STAT3 in H22 hepatocellular carcinoma cells. Oncol Rep. (2018) 40:2806-13. doi: 10.3892/or.2018.6651

46. Dong L, Hong H, Chen X, Huang Z, Wu W, Wu F. LINC02163 regulates growth and epithelial-to-mesenchymal transition phenotype via miR-5933p/FOXK1 axis in gastric cancer cells. Artif Cells Nanomed Biotechnol. (2018) 46:607-15. doi: 10.1080/21691401.2018.1464462

47. Matsumoto A, Thompson DC, Chen Y, Kitagawa K, Vasiliou V. Roles of defective ALDH2 polymorphism on liver protection and cancer development. Environ Health Prev Med. (2016) 21:395-402. doi: 10.1007/s12199-016-0579-2

48. Hou G, Chen L, Liu G, Li L, Yang Y, Yan H-X, et al. Aldehyde dehydrogenase2 (ALDH2) opposes HCC progression by regulating AMPK signaling. Hepatology. (2016) 65:1628-44. doi: 10.1002/hep.29006
49. Chen X, Legrand AJ, Cunniffe S, Hume S, Poletto M, Vaz B, et al. Interplay between base excision repair protein XRCC1 and ALDH2 predicts overall survival in lung and liver cancer patients. Cell Oncol. (2018) 41:527-39. doi: 10.1007/s13402-018-0390-8

50. Shih S, Huang YT, Yang HI. A multiple mediator analysis approach to quantify the effects of the ADH1B and ALDH2 genes on hepatocellular carcinoma risk. Genet Epidemiol. (2018) 42:394-404. doi: 10.1002/gepi.22120

Conflict of Interest: The authors declare that the research was conducted in the absence of any commercial or financial relationships that could be construed as a potential conflict of interest.

Copyright $\odot 2020$ Chen, Wang, Wang, Liu and Bai. This is an open-access article distributed under the terms of the Creative Commons Attribution License (CC BY). The use, distribution or reproduction in other forums is permitted, provided the original author(s) and the copyright owner(s) are credited and that the original publication in this journal is cited, in accordance with accepted academic practice. No use, distribution or reproduction is permitted which does not comply with these terms. 